\title{
Socio-Demographic Determinants of Family Planning Service Utilization among Women of Reproductive Age in Urban Communities of Imo State, Nigeria
}

\author{
Chukwuma B. Duru ${ }^{*}$, Obiageli F. Emelumadu², Anthony C. Iwu' ${ }^{1}$, Ikechi Ohanle ${ }^{3}$, \\ Chuka C. Agunwa ${ }^{4}$, Ernest Nwaigbo ${ }^{3}$, Emmanuel N. Ndukwu ${ }^{3}$ \\ ${ }^{1}$ Department of Community Medicine, Imo State University, Owerri, Nigeria \\ ${ }^{2}$ Department of Community Medicine, Nnamdi Azikiwe University, Awka, Nigeria \\ ${ }^{3}$ Department of Community Medicine, Imo State University Teaching Hospital, Orlu, Nigeria \\ ${ }^{4}$ Department of Community Medicine, University of Nigeria, Nsukka, Nigeria \\ Email: *duruchukwuma16@yahoo.com,drduruchukwuma@gmail.com
}

How to cite this paper: Duru, C.B., Emelumadu, O.F., Iwu, A.C., Ohanle, I., Agunwa, C.C., Nwaigbo, E. and Ndukwu, E.N. (2018) Socio-Demographic Determinants of Family Planning Service Utilization among Women of Reproductive Age in Urban Communities of Imo State, Nigeria. Open Access Library Journal, 5: e4627.

https://doi.org/10.4236/oalib.1104627

Received: April 27, 2018

Accepted: May 20, 2018

Published: May 23, 2018

Copyright $\odot 2018$ by authors and Open Access Library Inc.

This work is licensed under the Creative Commons Attribution International License (CC BY 4.0).

http://creativecommons.org/licenses/by/4.0/ (c) (i) Open Access

\begin{abstract}
Introduction: Many developing countries are characterized by rapid population growth, which is partly attributed to high fertility rates, high birth rates, accompanied by steady declines in death rates, low contraceptive use and high but declining mortality rate. AIM: To determine the factors influencing family planning method use among women of reproductive age in urban communities of Imo state, Nigeria. Methodology: The study was a community based descriptive cross-sectional study involving 560 women of reproductive age who met the inclusion criteria and were selected using the multistage sampling technique. The data were collected using a pretested, semi-structured and interviewer administered questionnaire and were analyzed using EPI-INFO version 3.2.1. A $p$-value $\leq 0.05$ was considered significant. Results: The mean age of the respondents was $32.7 \pm 7.7$ years with most of the women (99.1\%) being aware of family planning. The common methods known were; pills $(65.2 \%)$, injections (62.5\%), condoms (61.3\%) and natural family planning methods (49.5\%). The prevalence of ever use and current use of family planning was $54.1 \%$ and $35.1 \%$ respectively and the commonest method ever (36\%) and currently (26\%) used was the condom. The factors found to significantly influence family planning method use in the study were: age of women, religion, family size, number of male children, monthly income, being able to access and pay for family planning services without partners financial support, contraceptive knowledge, prior discussion of family planning with part-
\end{abstract}


ner, partner being in support of modern family planning methods use and previous use of any form of contraception. Conclusion: Most of the factors found to influence use can be modified by appropriate intervention programs. Thus there is need to institute these intervention programs by authorities concerned so as to increase utilization of services and reduce consequent problems.

\section{Subject Areas}

Sociology

\section{Keywords}

Determinants, Family Planning Methods, Urban Communities, Imo State

\section{Introduction}

According to World Health Organization expert Committee in 1971, family planning is defined as a way of thinking and living that is adopted voluntarily upon the basis of knowledge, attitudes and responsible decisions by individuals and couples in order to promote the health and welfare of the family group and thus contributes to the social development of the country [1]. It also refers to the conscious effort by couples to limit or space the number of children they want to have through the use of contraceptive methods [2]. Generally many developing countries like Nigeria are characterized by rapid population growth that is partly attributed to high fertility rate, high birth rates, accompanied by steady declines in death rates, low contraceptive use and high but declining mortality rate [3] [4] [5]. This rapid increase in population has an adverse effect on the national economy and also the increasing number of births has a deleterious effect on the health of the mother, which in turn hinders social and economic uplift of the family [6]. With natural growth rate of $2.4 \%$ and high fertility rate of 5.5 (rural, 6.2 , urban 4.7), the population of Nigeria is still rapidly increasing with an estimated population of 177.5 million in 2014 [7] [8] [9]. Going by this report, Nigeria is currently the $9^{\text {th }}$ largest country in the world and the most populous black nation [7] [8]. Worldwide, the 10 countries with the highest maternal mortality ratios (MMR) are in Africa of which Nigeria is one of them, and an estimated 14 percent of maternal deaths globally occur in Nigeria, despite the fact that Nigeria comprise only $2 \%$ of the world population [9] [10]. Despite these associated problems, the contraceptive prevalence rate in Nigeria has remained consistently low, steadying at $12.6 \%$ from 2003 to 2008 but only increased marginally to $15 \%$ for use of all methods in 2013 National Survey [9] [11] [12]. This is of grave concern because contraceptives had been found to effectively control population explosion if properly used. Due to this high fertility rate and consequent problems, one of the cardinal objectives of Nigeria National Policy on Population is to reduce the high level of fertility in the Country [13]. The guid- 
ing principle in achieving this objective is to emphasize the voluntary acceptance of family planning method in accordance with fundamental human rights and making them available at little or no cost [9] [13]. Several factors have been found to influence contraceptive use around the world and some of these factors have negative effects while others have positive effects on contraceptive use. Some of the factors found to influence use in some of the reviewed works were; age of women [4] [6] [14]-[19], marital status [14] [16] [19] [20] [21], religion [4] [6] [15] [17] [19] [20] [22], level of education of women [5] [6] [14] [16] [17] [18] [19] [21]-[28], level of knowledge about family planning [4] [5] [15] [23] [24], socio economic status of women (monthly income, occupation, ownership of household items and wealth index) [4] [5] [17] [18] [19] [20] [25], fertility related issues, (parity, family size, number of males, age at marriage, and years of marriage) [4] [6] [17] [18] [19] [21] [26] [27], place of residence (rural or urban)/region of the country of respondent [18] [19] [20] [27], tribe of respondents [16] [19], family setting (monogamy or polygamy) [22], and attitude towards family planning [24]. Issues concerning place of service like; quality of service rendered, proximity of service point, friendliness of care providers [4] and availability of family planning service [24] were also found to influence the use of family planning methods.

Others factors reported to have influenced contraceptive use were issues related to their male partners like; partners/spousal approval of use or support [4] [15] [23] [27] [28], prior discussion with partner [27], husbands occupation [28], and level of education [18] [27].

Thus this study aimed to determine the factors influencing family planning method use among women of reproductive age in urban communities of Imo state, Nigeria.

\section{Methodology}

\subsection{Description of Study Area}

Imo State is one of the 36 States of Nigeria in the South Eastern Region of the country. It has a total population of about 3.93 million people, comprising more males than females (2.03 million and 1.9 million people respectively), with population growth rate of 2.89 from 1991-1998 and 3.0 from 1999-2005. The majority of the people living in the State are Igbos [12]. The State has 27 Local Government Areas of which 5 are urban as designated by the National Population Commission (NPC) [12]. The major occupation of the people in the urban areas is trading, artisan and public service. The main religion of the people is Christianity. Owerri Municipal Local Government Area is an Urban Local Government Area and the state capital.

\subsection{Study Design/Study Population/Inclusion Criteria}

The study was a cross-sectional survey of the factors influencing family planning services use among women of reproductive age group (15 - 49 years) in urban 
Communities in Imo state. The study population consisted women of reproductive age group in the selected study areas of the state. All women of reproductive age (15 - 49 years) whether married or single were included in this survey. For an individual to be selected she must have been resident in the enumeration areas selected for the study for at least a period of one year prior to commencement of research.

\subsection{Minimum Sample Size Estimation}

Using the Cochran sample size formula for calculating minimum sample size in populations greater than 10,000 and proportions of women in reproductive age group in South East Nigeria who are currently using any form of contraceptive method among urban dwellers from a previous study (15.2\%) [14].

$$
n=Z^{2} p q / d^{2}
$$

$n=$ Sample size to be estimated, $p=$ Proportion of women within the reproductive age currently using any form of contraceptive method (15.2\%) [14], $Z=$ Standard normal deviate corresponding to $95 \%$ significance level $\approx 1.96, d=$ level of precision desired for the study set at 0.05 . The total sample size of 560 was used for this study.

\subsection{Sampling Technique}

The sampling technique used for this study was multistage sampling technique. The first stage involved the selection of the Local Government Area that was used for the study from the 27 Local Government Areas in the State. The Local Government Areas (LGA's) were grouped into 2 categories. Category A, were made up of 5 urban Local Government Areas; and category B, consisting of 22 rural Local Government Areas. From category A, Owerri Municipal Local Government Area was selected by simple random sampling technique, using balloting. The second stage involved the selection of the primary sampling units from the LGA's. The enumeration areas (EA's) which are geographic clusters that have been clearly demarcated by the National Population Commission (NPC), served as the primary sampling units. Then using simple random sampling technique, ten EA's were selected out of 750 EA's in Owerri Municipal.

The third stage involved the selection of the respondents that were interviewed. From the LGA, 560 women in the reproductive age group (15 - 49 years) were recruited for the survey, thus a total of 57 respondents per EA were recruited. In each of the selected EA's, a random starting point was determined in the field by the supervisor using a community landmark such as village square, church, or a mosque, market, school or streets and movement was in a clockwise direction. Eligible respondents were consecutively recruited and interviewed until the required sample size for the selected EA was achieved.

In any EA where the required sample size could not be obtained, simple random sampling was used to select another EA outside those previously selected and respondents studied until the required size for that EA is completed. Only 
one eligible respondent per household was interviewed during the survey. If a household had more than one eligible respondent, only one was randomly selected by simple balloting.

\subsection{Data Collection Process, Techniques and Analysis}

There was proper community entry, sensitization and mobilization. A semi-structured, pretested, interviewer administered questionnaire for women of reproductive age group was used. The questionnaire was pretested for comprehensibility, appropriateness of language, sensitivity of questions, and average duration for administration. The questionnaire was divided into 2 major sections, the first section was designed to obtain the socio-demographic characteristics of the respondents and the second section was designed to access the knowledge, awareness, practice and use of family planning services. This was to ensure validity and reliability of the study. Data collected was cleaned and validated manually, while a computer software package (EPI INFO version 3.3.2) was used for data entry and analysis. Frequencies and percentages of relevant variables were generated and bivariate analysis conducted using chi-square. For the purpose of this study; respondents who could not mention any family planning method were categorized as having poor knowledge, any correct mention of any two (2) or less was classified as fair knowledge and correct mention of any three (3) or more was classified as having good knowledge of family planning methods. A $p$-value $<0.05$ was considered.

\subsection{Ethical Consideration}

Ethical clearance was obtained from the Ethics Committee of the Nnamdi Azikiwe University Teaching Hospital Nnewi (NAUTHEC) before the commencement of the study. In addition before the questionnaires were administered, the concept of the study was carefully explained to each participant and consent obtained from all the respondents. They were also assured of confidentiality and that participation was optional. All authors hereby declare that the study was performed in accordance with the ethical standards laid down in the 1964 Declaration of Helsinki.

\section{Results}

Table 1 shows that mean age of the respondents was $32.7 \pm 7.7$ years with majority of them, 365 (65.9\%) being currently married, had secondary 295, (52.3\%) or tertiary 244, (43.6\%) education, were of catholic Christian denomination, 251 (44.8\%), employed, 410 (73.2\%), of Igbo extraction, 550 (98.2\%) and were in monogamous unions, $390(92.2 \%)$. Average family size and number of male children were $3.5 \pm 0.8$ and $1.5 \pm 0.4$ respectively with a median personal monthly income of 20,320 naira (68 USD). Most of the married women, 350 $(82.7 \%)$ were currently staying together with their husbands' and majority of their husbands, 413 (97.6\%) were employed, had secondary 216 (51.1\%) or tertiary education, 174 (41.1\%). 
Table 1. Socio-demographic characteristics of respondents.

\begin{tabular}{|c|c|c|}
\hline Variable & Frequency $(n=560)$ & Percentage \\
\hline \multicolumn{3}{|l|}{ Age group (yrs) } \\
\hline $15-19$ & 14 & 2.5 \\
\hline $20-24$ & 66 & 11.8 \\
\hline $25-29$ & 143 & 25.5 \\
\hline $30-34$ & 109 & 19.5 \\
\hline $35-39$ & 92 & 16.4 \\
\hline $40-44$ & 94 & 16.8 \\
\hline $45-49$ & 42 & 7.5 \\
\hline \multicolumn{3}{|l|}{ Mean age $=32.7 \pm 7.7$} \\
\hline \multicolumn{3}{|l|}{ Marital status } \\
\hline Currently married & 365 & 65.9 \\
\hline Single & 98 & 17.5 \\
\hline Co-habiting & 58 & 10.4 \\
\hline Previously married & 39 & 7.2 \\
\hline \multicolumn{3}{|l|}{ Educational status } \\
\hline Primary & 23 & 4.1 \\
\hline Secondary & 293 & 52.3 \\
\hline Tertiary & 244 & 43.6 \\
\hline \multicolumn{3}{|l|}{ Religion } \\
\hline Catholics & 251 & 44.8 \\
\hline Pentecostal & 178 & 31.8 \\
\hline Orthodox & 114 & 20.4 \\
\hline Traditional religion & 17 & 3.0 \\
\hline \multicolumn{3}{|l|}{ Employment status } \\
\hline Employed & 410 & 73.2 \\
\hline Unemployed & 150 & 26.8 \\
\hline \multicolumn{3}{|l|}{ Tribe } \\
\hline Igbo & 550 & 98.2 \\
\hline Non-Igbo & 10 & 1.8 \\
\hline \multicolumn{3}{|c|}{ Family type (for currently married and cohabiting) $(n=423)$} \\
\hline Monogamy & 390 & 92.2 \\
\hline Polygamy & 33 & 7.8 \\
\hline \multicolumn{3}{|c|}{ No. of living children $(n=462)$} \\
\hline None & 65 & 14.5 \\
\hline $1-4$ & 281 & 60.8 \\
\hline$>4$ & 114 & 26.9 \\
\hline
\end{tabular}




\section{Continued}

Mean $=3.5 \pm 0.8$

No. of males $(n=462)$

$\begin{array}{ccc}\text { None } & 130 & 28.1 \\ 1-2 & 239 & 51.7 \\ 3-4 & 77 & 16.7 \\ >4 & 16 & 3.5\end{array}$

Mean $=1.5 \pm 0.4$

Monthly personal income

\begin{tabular}{|c|c|c|}
\hline$<10,000$ & 113 & 20.2 \\
\hline $10,000-19,999$ & 202 & 36.1 \\
\hline $20,000-29,999$ & 120 & 21.4 \\
\hline $30,000-39,999$ & 49 & 8.8 \\
\hline More than or equal to 40,000 & 42 & 7.5 \\
\hline Unsure & 34 & 6.0 \\
\hline \multicolumn{3}{|c|}{ Median income $=20,320$ Naira $(\$ 102 U S D)$} \\
\hline \multicolumn{3}{|c|}{ Currently staying together with husband $(n=423)$} \\
\hline Yes & 350 & 82.7 \\
\hline No & 73 & 17.3 \\
\hline \multicolumn{3}{|c|}{ Husbands level of education $(n=423)$} \\
\hline$\leq$ Primary & 33 & 7.8 \\
\hline Secondary & 216 & 51.1 \\
\hline Tertiary & 174 & 41.1 \\
\hline \multicolumn{3}{|l|}{ usbands occupation ( $n=423$ ) } \\
\hline Employed & 413 & 97.6 \\
\hline Unemployed & 10 & 2.4 \\
\hline
\end{tabular}

Table 2 shows the level of awareness and knowledge about family planning amongst women. Almost all the women, 555 (99.1\%), have had about family planning and the common methods mentioned were; pills, (63.2\%) injections, (62.5\%), condoms, $(61.3 \%$ and natural family planning methods/rhythm, (49.5\%). Majority of the women, $450(81.1 \%)$ had a good knowledge about family planning methods.

Table 3 shows that contraceptive knowledge increases significantly with increase in educational attainment of respondents with those that had tertiary education having better contraceptive knowledge than others, $\left(X^{2}=25.30, p=\right.$ 0.001) (Table 3).

Table 4 shows distribution of respondents' views on issues concerning male involvement in family planning. Slightly above one third of women (28.4\%) agreed that their partners did something to delay pregnancy though majority of 
Table 2. Awareness and knowledge about family planning.

\begin{tabular}{|c|c|c|}
\hline Variable & Frequency & Percentage \\
\hline \multicolumn{3}{|c|}{ Aware about family planning $(n=560)$} \\
\hline Yes & 555 & 99.1 \\
\hline No & 5 & 0.9 \\
\hline \multicolumn{3}{|c|}{${ }^{*}$ Family planning methods known $(\mathrm{n}=555)$} \\
\hline Pills & 351 & 63.2 \\
\hline Injections & 347 & 62.5 \\
\hline Condoms & 340 & 61.3 \\
\hline Natural Method/Rhythm & 275 & 49.5 \\
\hline IUCD Loops & 246 & 43.3 \\
\hline Withdrawal Methods & 144 & 26.0 \\
\hline Sterilization Methods & 143 & 25.8 \\
\hline Abstinence & 95 & 16.9 \\
\hline Diaphragm & 67 & 11.9 \\
\hline Foam/Jelly Spermicide & 42 & 7.5 \\
\hline Exclusive Breastfeeding & 32 & 5.7 \\
\hline Implants & 20 & 3.6 \\
\hline Traditional Methods & 7 & 1.3 \\
\hline \multicolumn{3}{|l|}{ Level of knowledge $(n=555)$} \\
\hline Poor(no method mentioned) & 14 & 2.5 \\
\hline Fair (1 - 2 methods) & 91 & 16.4 \\
\hline Good ( $\geq 3$ methods) & 450 & 81.1 \\
\hline
\end{tabular}

** = multiple response.

Table 3. Educational attainment and contraceptive knowledge.

\begin{tabular}{|c|c|c|c|c|c|}
\hline \multirow{2}{*}{ Educational level } & \multicolumn{3}{|c|}{ Level of contraceptive knowledge } & \multirow{2}{*}{ Total (\%) } & \multirow{2}{*}{ Chi-square/ $p$-value } \\
\hline & Poor (\%) & Fair (\%) & Good (\%) & & \\
\hline Primary & $2(5.8)$ & $10(26.5)$ & $23(67.7)$ & $35(100)$ & \\
\hline Secondary & $8(2.9)$ & $59(22.0)$ & $210(75.1)$ & $279(100)$ & $X^{2}=25.30$ \\
\hline Tertiary & $4(1.7)$ & $22(9.0)$ & $217(89.3)$ & $243(100)$ & $P=0.001^{*}$ \\
\hline Total & $14(2.5)$ & $91(16.6)$ & $450(80.9)$ & $555(100)$ & \\
\hline
\end{tabular}

* = significant.

Table 4. Distributions of Respondents views on issues concerning male involvement in family planning.

\begin{tabular}{ccc}
\hline Variable & Frequency $(\mathbf{n}=\mathbf{5 6 0})$ & Percentage \\
\hline \multicolumn{2}{c}{ Whether husband/ } & partner do anything to delay pregnancy \\
Yes & 215 & 38.4 \\
No & 345 & 61.6 \\
Can you access and pay for family planning services without your partners financial support \\
Yes & 296 & 52.9 \\
No & 264 & 47.1 \\
\hline
\end{tabular}




\section{Continued}

Is your Husband's/Partner's opinion important in family planning use

$\begin{array}{lcc}\text { Yes } & 519 & 92.7 \\ \text { No } & 41 & 7.3\end{array}$

Can you decide use family planning without their Husband/ Partner's Consent

$\begin{array}{lll}\text { Yes } & 102 & 18.2\end{array}$

$\begin{array}{llr}\text { No } & 458 & 81.9\end{array}$

Have you ever discussed Family Planning with Husbandor Partner

$\begin{array}{lll}\text { Yes } & 231 & 41.3\end{array}$

$\begin{array}{lll}\text { No } & 329 & 58.7\end{array}$

Husbands/Partners is/are in support Modern Family Planning Methods

$\begin{array}{lll}\text { Yes } & 250 & 44.6\end{array}$

$\begin{array}{lll}\text { No } & 301 & 55.4\end{array}$

them, (92.7\%) responded that their partner opinion was important in family planning use. Majority of the women (81.9\%) said that they cannot decide to use family planning without their partners consent while a lower proportion of them, $(41.3 \%)$ claimed to have ever discussed family planning issues with their spouses. Less than half of their partners, (44.6\%) were in support of modern family planning methods.

Figure 1 shows that the prevalence of contraceptive ever used was $54.1 \%$ while current use was $35.2 \%$.

Table 5 and Table 6 shows that contraceptives methods commonly ever used were; condoms (36.0\%), NFP/rhythm (27.7\%), pills (25.0\%), and injections $(22.3 \%)$ while the common methods currently used were; condoms (26.0\%), NFP/Rhythm (24.5\%), withdrawal methods, (19.9\%) and IUCD (19.4\%). Commonest reason for choice of contraception used was; it is convenient, (49.5\%), followed by it was recommended by healthcare professionals, $(39.8 \%)$ and it is effective $(35.2 \%)$, while the common reason for not using any form of contraception were; I want more children (47.4\%), I am pregnant (24.1\%), and fear of health problems/side effect (25.5\%) (Table 5 and Table 6).

Table 7 demonstrates that age of the women influenced contraceptive ever use significantly, $\left(X^{2}=40.530, p=0.000\right)$, showing an increasing likelihood of ever used with increasing age of respondents, those 45 - 49 years of age had the highest likelihood to have ever used any form of contraception $(\mathrm{OR}=18.64 ; 3.97$ - 83.22) than women in the other age groups. Employed or working women were more likely to have ever used any form of contraception in their life time than their unemployed counterparts, $\left(X^{2}=10.801, P=0.000, \mathrm{OR}=0.53 ; 0.36-0.78\right)$. Those with greater than four children, $\left(X^{2}=54.144, p=0.000\right.$, OR: $22.55 ; 10.00-$ $50.84)$ and 3 - 4 male children, $\left(X^{2}=49.910, p=0.000\right.$, OR: $\left.12.50 ; 6.36-24.60\right)$ were more likely to have used any form of contraception than their counterparts. Level of contraceptive knowledge influenced ever use of any form of 


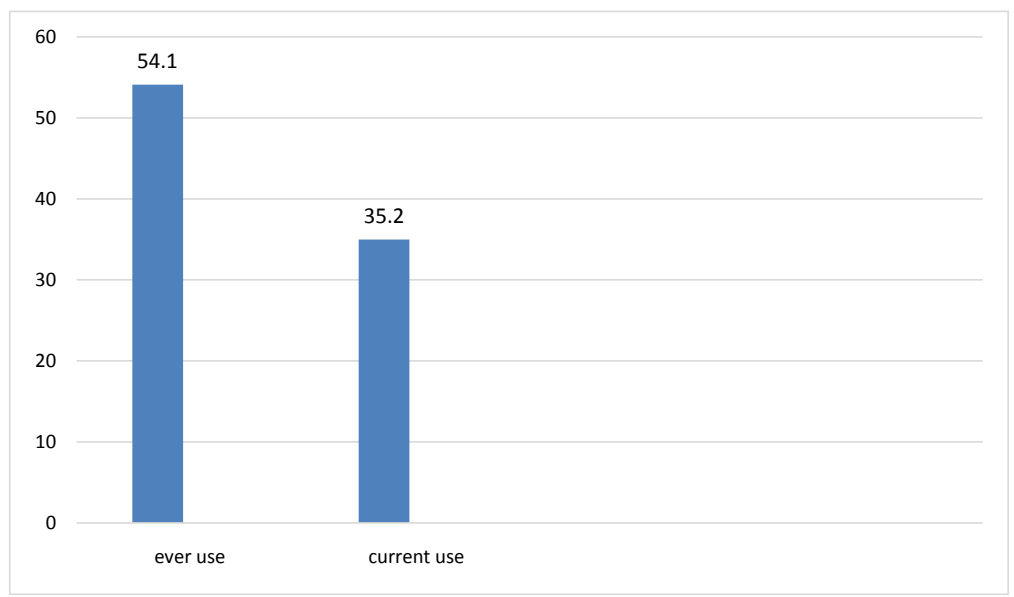

Figure 1. Prevalence of contraceptive use among respondents.

Table 5. Contraceptive methods ever used and currently being used among respondents.

\begin{tabular}{ccc}
\hline$*$ Contraceptive methods & Ever used $(\%) \mathbf{n}=\mathbf{3 0 3}$ & Currently using $(\%) \mathbf{n}=\mathbf{1 9 6}$ \\
\hline Condom & $108(36.0)$ & $51(26.0)$ \\
Rhythm & $83(27.7)$ & $48(24.5)$ \\
Pills & $75(25.0)$ & $24(12.2)$ \\
Injections & $67(22.3)$ & $34(17.4)$ \\
Withdrawal method & $67(22.3)$ & $39(19.9)$ \\
IUCD/loop & $58(19.3)$ & $38(19.4)$ \\
Breastfeeding & $44(14.7)$ & $15(7.7)$ \\
Abstinence & $20(6.6)$ & $24(12.2)$ \\
Tubal ligation & $15(5.0)$ & $15(7.7)$ \\
Others & $16(5.3)$ & $14(7.1)$ \\
\hline
\end{tabular}

Others $=$ diaphragm, cervical caps, vaginal foams/gels/spermicides and traditional methods. ${ }^{*}=$ multiple response.

Table 6. Reasons for current contraceptive method use and non-use.

\begin{tabular}{ccc}
\hline Variable & Frequency & Percentage \\
\hline${ }^{*}$ Reasons for contraceptive & method use $(\mathbf{n}=196)$ & \\
\hline It is convenient & 97 & 49.5 \\
Recommended by Health Care Professional & 78 & 39.8 \\
It is effective & 69 & 35.2 \\
Easy to use & 47 & 24.0 \\
Has less side effects & 33 & 16.8 \\
It is cheap & 29 & 14.8 \\
Recommended by friends and relatives & 19 & 9.7 \\
Can be used without partners knowledge & 15 & 7.7 \\
Is easily reversible & 14 & 7.1 \\
\hline
\end{tabular}




\section{Continued}

\begin{tabular}{ccc}
\hline \multicolumn{2}{c}{ Main reasons for not currently using any method $(\mathrm{n}=364)$} \\
Want more children & 171 & 47.4 \\
Health Problems/side effects & 93 & 25.5 \\
Pregnant & 88 & 24.1 \\
Not married & 57 & 15.8 \\
Religious reasons & 42 & 11.6 \\
Not sexually active & 39 & 10.8 \\
Infrequent sex & 17 & 4.7 \\
Not convenient & 14 & 3.9 \\
Don't know method/source & 11 & 3.1 \\
Too costly & 4 & 1.1 \\
\hline
\end{tabular}

** $=$ Multiple response.

Table 7. Factors influencing ever use of contraceptives among respondents.

\begin{tabular}{|c|c|c|c|c|c|}
\hline \multirow{2}{*}{ Variable } & \multicolumn{3}{|c|}{ Contraceptive ever use $(n=560)$} & \multirow{2}{*}{$\begin{array}{c}\text { Chi-square } \\
p \text {-value }\end{array}$} & \multirow{2}{*}{ OR $(95 \% C I)$} \\
\hline & Yes (\%) & No (\%) & Total (\%) & & \\
\hline \multicolumn{6}{|l|}{ Age (yrs) } \\
\hline $15-19$ & $2(21.4)$ & $11(78.6)$ & $14(100)$ & & 1.00 \\
\hline $20-24$ & $24(36.4)$ & $42(63.6)$ & $66(100)$ & 40.530 & $2.10(0.53,8.26)$ \\
\hline $25-29$ & $67(46.9)$ & $76(53.1)$ & $143(100)$ & $p<0.0001^{*}$ & $3.23(0.87,12.07)$ \\
\hline $30-34$ & $55(50.5)$ & $54(49.5)$ & $109(100)$ & & $3.74(0.99,14.13)$ \\
\hline $35-39$ & $49(53.3)$ & $43(46.7)$ & $92(100)$ & & $4.18^{\star}(1.09,15.97)$ \\
\hline $40-44$ & $70(74.5)$ & $24(25.5)$ & $94(100)$ & & $10.69^{*}(2.75,41.59)$ \\
\hline $45-49$ & $35(83.3)$ & $7(16.7)$ & $42(100)$ & & $18.64^{*}(3.97,83.33)$ \\
\hline Total & $303(54.1)$ & $257(45.9)$ & $560(100)$ & & \\
\hline \multicolumn{6}{|l|}{ Marital status } \\
\hline Currently married & $197(54.0)$ & $168(46.0)$ & $365(100)$ & & 1.00 \\
\hline Never married & $45(45.9)$ & $53(54.1)$ & $98(100)$ & 0.103 & $0.72(0.46,1.13)$ \\
\hline Cohabiting & $37(63.8)$ & $21(36.2)$ & $58(100)$ & $P=0.748$ & $1.50(0.85,2.67)$ \\
\hline Previously married & $24(61.5)$ & $15(38.5)$ & $39(100)$ & & $1.36(0.69,2.68)$ \\
\hline Total & $303(54.1)$ & $257(45.9)$ & $560(100)$ & & \\
\hline \multicolumn{6}{|l|}{ Educational status } \\
\hline$\leq$ Primary & $11(47.8)$ & $12(52.2)$ & $23(100)$ & & 1.00 \\
\hline Secondary & $154(52.6)$ & $139(47.4)$ & $293(100)$ & 0.022 & $1.21(0.52,2.83)$ \\
\hline Tertiary & $138(56.6)$ & $106(43.4)$ & $244(100)$ & $P=0.881$ & $1.42(0.59,3.42)$ \\
\hline Total & $303(54.1)$ & $257(45.9)$ & $560(100)$ & & \\
\hline \multicolumn{6}{|l|}{ Religion } \\
\hline Catholics & $132(52.6)$ & $119(47.2)$ & $251(100)$ & & 1.00 \\
\hline Pentecostal & $88(49.4)$ & $90(50.6)$ & $178(100)$ & 2.029 & $0.88(0.60,1.29)$ \\
\hline Orthodox & $74(64.9)$ & $40(35.1)$ & $114(100)$ & $P=0.154$ & $1.67^{\star}(1.06,2.64)$ \\
\hline Others & $9(54.9)$ & $8(47.1)$ & $17(100)$ & & $1.01(0.38,2.71)$ \\
\hline
\end{tabular}


Continued

\begin{tabular}{|c|c|c|c|c|c|}
\hline Total & $303(54.1)$ & $257(45.9)$ & $560(100)$ & & \\
\hline \multicolumn{6}{|c|}{ Employment status } \\
\hline Employed & $239(58.3)$ & $171(41.7)$ & $410(100)$ & 10.799 & 1.00 \\
\hline Unemployed & $64(42.7)$ & $86(57.3)$ & $150(100)$ & $p<0.0001^{*}$ & $0.53^{*}(0.37,0.78)$ \\
\hline Total & $303(54.1)$ & $257(45.9)$ & $560(100)$ & & \\
\hline \multicolumn{6}{|c|}{ Family size(ever married and cohabiting) $n=462$} \\
\hline None & $10(14.9)$ & $57(85.1)$ & $67(100)$ & & 1.00 \\
\hline $1-4$ & $157(55.9)$ & $124(44.1)$ & $281(100)$ & 54.144 & $7.22 *(3.54,14.71)$ \\
\hline$>4$ & $91(79.8)$ & $23(20.2)$ & $114(100)$ & $p<0.0001^{*}$ & $22.55^{*}(10.00,50.84)$ \\
\hline Total & $258(55.8)$ & $204(44.2)$ & $462(100)$ & & \\
\hline \multicolumn{6}{|c|}{ No. of males(ever married and cohabiting) $n=462$} \\
\hline None & $27(20.8)$ & $103(79.2)$ & $130(100)$ & & 1.00 \\
\hline $1-2$ & $102(67.8)$ & $77(32.2)$ & $239(100)$ & 49.906 & $8.03^{*}(4.85,13.27)$ \\
\hline $3-4$ & $59(76.6)$ & $18(23.4)$ & $77(100)$ & $p<0.0001^{*}$ & $12.50^{*}(6.36,24.60)$ \\
\hline$>4$ & $10(62.5)$ & $6(37.5)$ & $16(100)$ & & $6.36^{*}(2.12,19.05)$ \\
\hline Total & $258(55.8)$ & $204(44.2)$ & $462(100)$ & & \\
\hline \multicolumn{6}{|c|}{ Contraceptive knowledge } \\
\hline Poor & $1(7.1)$ & $13(92.9)$ & $14(100)$ & & 1.00 \\
\hline Fair & $25(27.5)$ & $66(72.5)$ & $91(100)$ & 48.248 & $4.92(0.61,39.54)$ \\
\hline Good & $278(61.8)$ & $172(38.2)$ & $450(100)$ & $p<0.0001^{*}$ & $21.01^{*}(2.72,162.05)$ \\
\hline Total & $303(54.6)$ & $252(45.4)$ & $555(100)$ & & \\
\hline
\end{tabular}

* = statistically significant.

contraception significantly, $\left(X^{2}=48.250, p=0.000\right)$ and those with high or good knowledge were the most likely to have used any form of contraception than their counterparts with low knowledge about contraceptive methods (OR: 21.01; 2.72 - 162.05). Generally, religion had no significant influence on ever use among the women $(P>0.05)$ but those of orthodox domination were more likely to have ever used any form of contraception than their counterparts in other dominations (OR: 1.68; 1.06 - 2.64). Marital and educational status of women had no significant effect on ever use of any form of contraception, $(p>0.05)$.

Table 8 shows factors influencing current use of contraceptives among women in urban communities of Imo State, Nigeria. Age of women was found to significantly influence current use of any form of contraception $\left(X^{2}=21.740, p=\right.$ 0.001 ) with women 40 - 44 years, (OR: 4.83; 1.05 - 35.27) being more likely to use than their younger counterparts. Religious denominations attended by the women played a significant role $\left(X^{2}=5.72, p=0.017\right)$ with women in orthodox denomination (OR: 1.86; 1.18 - 2.93) being the most likely to be using any form of contraception than their other counterparts in other denominations. Family size, $\left(\mathrm{X}^{2}=35.710, p=0.000\right)$ and number of males a woman had, $\left(\mathrm{X}^{2}=39.90 . p\right.$ $=0.000)$ had significant influence on current use of contraception with those of the family sizes greater than four, (OR: $3486 ; 8.14$ - 149.26) and males greater 
Table 8. Factors influencing current use of contraceptives among respondents.

\begin{tabular}{|c|c|c|c|c|c|}
\hline \multirow{2}{*}{ Variable } & \multicolumn{3}{|c|}{ Current contraceptive use } & \multirow{2}{*}{$\begin{array}{c}\text { Chi-square } \\
p \text {-value }\end{array}$} & \multirow{2}{*}{ OR ( $95 \mathrm{CI})$} \\
\hline & Yes (\%) & No (\%) & Total (\%) & & \\
\hline \multicolumn{6}{|l|}{ Age (yrs) } \\
\hline $15-19$ & $14(14.3)$ & $12(85.7)$ & $14(100)$ & & 1.00 \\
\hline $20-24$ & $17(25.8)$ & $49(74.2)$ & $66(100)$ & 21.740 & $2.08(0.42,10.26)$ \\
\hline $25-29$ & $43(30.1)$ & $100(69.9)$ & $143(100)$ & $p=0.001$ & $2.58(0.55,12.02)$ \\
\hline $30-34$ & $32(29.4)$ & $77(70.6)$ & $109(100)$ & & $2.49(0.53,11.78)$ \\
\hline $35-39$ & $34(37.0)$ & $58(63.0)$ & $92(100)$ & & $3.52(0.74,16.67)$ \\
\hline $40-44$ & $49(52.1)$ & $45(47.9)$ & $94(100)$ & & $6.53^{*}(1.39,30.80)$ \\
\hline $45-50$ & $19(45.2)$ & $23(54.8)$ & $42(100)$ & & $4.83^{\star}(1.05,35.27)$ \\
\hline Total & $196(35.0)$ & $364(65.0)$ & $560(100)$ & & \\
\hline \multicolumn{6}{|l|}{ Marital status } \\
\hline Currently married & $124(34.0)$ & $241(66.0)$ & $365(100)$ & & 1.00 \\
\hline Never married & $37(37.8)$ & $61(62.2)$ & $98(100)$ & 0.384 & $1.18(0.74,1.87)$ \\
\hline Cohabiting & $25(43.1)$ & $33(56.9)$ & $58(100)$ & $p=0.536$ & $1.47(0.84,2.59)$ \\
\hline Previously married & $10(25.6)$ & $29(74.4)$ & $39(100)$ & & $0.67(0.32,1.42)$ \\
\hline Total & $196(35.0)$ & $364(65.0)$ & $560(100)$ & & \\
\hline \multicolumn{6}{|l|}{ Educational status } \\
\hline$\leq$ Primary & $7(30.4)$ & $16(69.6)$ & $23(100)$ & & 1.00 \\
\hline Secondary & $104(35.5)$ & $189(64.5)$ & $293(100)$ & 0.240 & $1.28(0.50,3.16)$ \\
\hline Tertiary & $85(34.8)$ & $159(65.2)$ & $244(100)$ & $p=0.624$ & $1.22(0.48,3.06)$ \\
\hline Total & $196(35.0)$ & $364(100)$ & $560(100)$ & & \\
\hline \multicolumn{6}{|l|}{ Religion } \\
\hline Catholics & $78(31.1)$ & $173(68.9)$ & $251(100)$ & & 1.00 \\
\hline Pentecostal & $59(33.1)$ & $119(66.9)$ & $178(100)$ & 5.717 & $1.10(0.73,1.67)$ \\
\hline Orthodox & $52(45.6)$ & $62(54.4)$ & $114(100)$ & $p=0.017^{*}$ & $1.86^{*}(1.18,2.93)$ \\
\hline Others & $7(41.2)$ & $10(58.8)$ & $17(100)$ & & $1.55(0.57,4.23)$ \\
\hline Total & $196(35.0)$ & $364(65.0)$ & $560(100)$ & & \\
\hline \multicolumn{6}{|l|}{$\begin{array}{l}\text { Employment } \\
\text { status }\end{array}$} \\
\hline Employed & $152(37.1)$ & $258(62.9$ & $410(100)$ & 2.561 & 1.00 \\
\hline Unemployed & $44(29.3)$ & $106(70.7)$ & $150(100)$ & $p=0.109$ & $0.71(0.47,1.06)$ \\
\hline Total & $196(35.0)$ & $364(65.0)$ & $560(100)$ & & \\
\hline \multicolumn{6}{|c|}{ Family size(ever married and cohabiting) $n=462$} \\
\hline None & $2(3.0)$ & $65(97.0)$ & $67(100)$ & & 1.00 \\
\hline $1-4$ & $98(34.9)$ & $183(65.1)$ & $281(100)$ & 35.705 & $17.40^{\star}(4.17,72.61)$ \\
\hline$>4$ & $59(51.8)$ & $55(48.2)$ & $114(100)$ & $p<0.0001^{*}$ & $34.86^{*}(8.14,149.26)$ \\
\hline Total & $159(34.4)$ & $303(65.6)$ & $462(100)$ & & \\
\hline \multicolumn{6}{|c|}{ No. of males (ever married and cohabiting) $n=462$} \\
\hline None & $12(7.7)$ & $118(92.3)$ & $130(100)$ & & 1.00 \\
\hline $1-2$ & $96(40.2)$ & $143(59.8)$ & $239(100)$ & 39.900 & $6.60^{*}(3.45,12.62)$ \\
\hline $3-4$ & $41(53.2)$ & $36(46.8)$ & $77(100)$ & $\mathrm{p}<0.0001^{*}$ & $11.20^{*}(5.32,23.56)$ \\
\hline$>4$ & $10(62.5)$ & $6(37.5)$ & $16(100)$ & & $16.38^{*}(5.07,52.99)$ \\
\hline
\end{tabular}




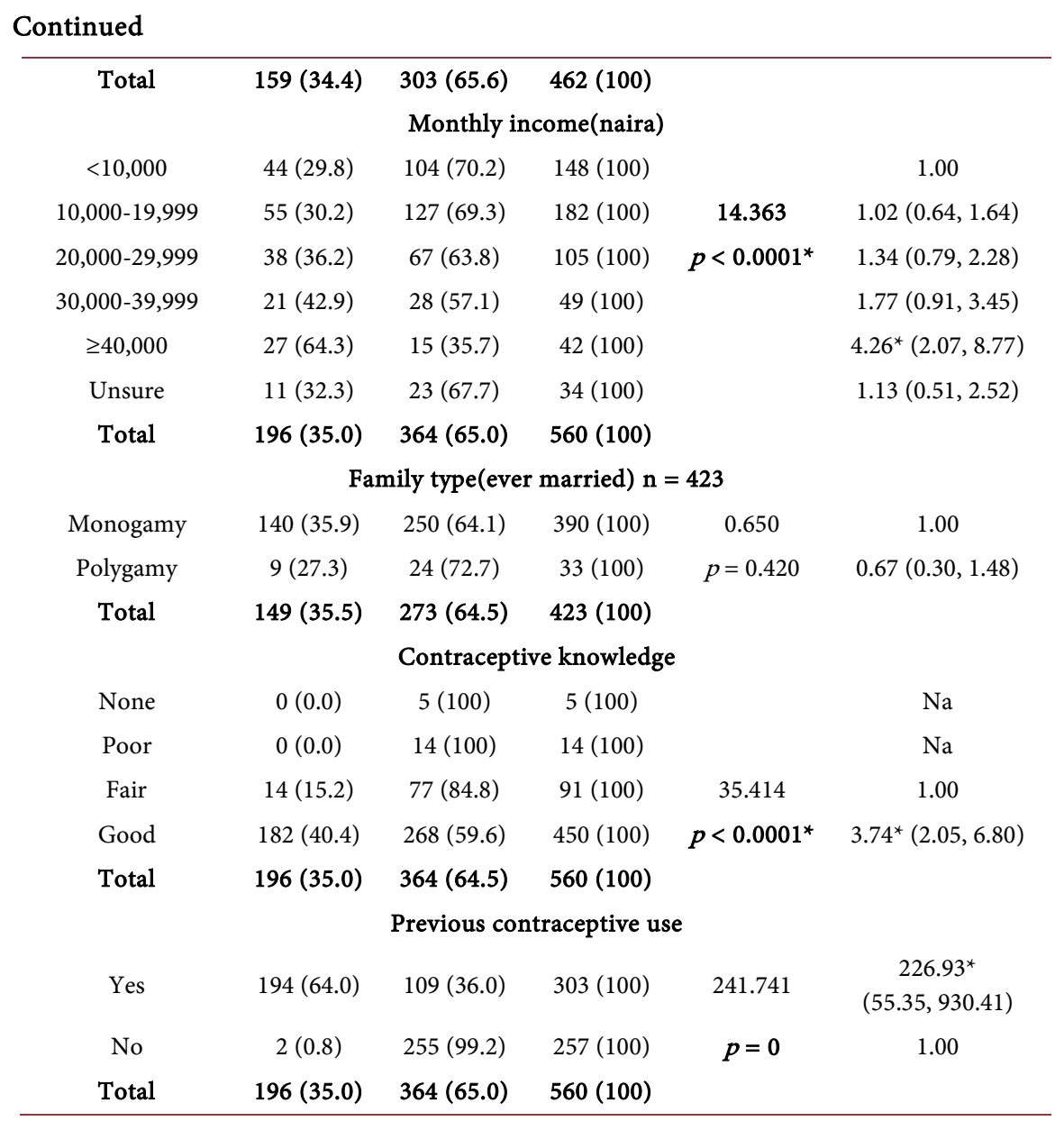

* = statistically significant, $\mathrm{Na}=$ not applicable.

than four (OR: 16.39; 5.07 - 52.99) being more likely to be using any form of contraception than their counterparts. Monthly income of the respondents significantly influenced current use of contraception, $\left(X^{2}=14.360, p=0.000\right)$ with those earning 40,000 Naira (110 USD) and above being more likely to be using any contraception than the other counterparts with lower income, (OR: 4.26; 2.07 - 8.77). Women with good or high knowledge about contraceptive methods are more likely to be using any form of contraception than their counterparts with lower contraceptive knowledge $\left(X^{2}=35.440, p=0.000\right.$, OR: 3.74; 2.05 6.80). Those who had used any form of contraception previously were more likely to currently use, $\left(X^{2}=241.741, p=0\right.$, OR: 226.93; 55.35 - 930.41). Marital status, educational level of women, employment status, and family type did not play any significant role in influencing contraceptive use among the women, $(p>0.05)$.

Table 9 shows the influence of male associated factors on current use of any form of contraception among the women. Women who have ever discussed family planning with their partners, $\left(X^{2} 148.230, p=0.0000\right.$, OR: 10.90; 7.24 16.43), those whose partners were in favour of modern family planning methods, $\left(X^{2}=73.180, p=0.000\right.$, OR: $\left.4.94 ; 3.41-7.24\right)$ and women who could access and 
Table 9. Influence of male associated factors on current use of contraceptives among respondents.

\begin{tabular}{|c|c|c|c|c|c|}
\hline \multirow{2}{*}{ Variable } & \multicolumn{3}{|c|}{ Current contraceptive use } & \multirow{2}{*}{$\begin{array}{l}\text { Chi-square/ } \\
p \text {-value }\end{array}$} & \multirow{2}{*}{ OR $(95 \% \mathrm{CI})$} \\
\hline & Yes (\%) & No (\%) & Total (\%) & & \\
\hline \multicolumn{6}{|c|}{ Husbands level of education (ever married) $n=462$} \\
\hline$\leq$ Primary & $11(33.3)$ & $22(66.7)$ & $33(100)$ & & 1.00 \\
\hline Secondary & $79(36.6)$ & $137(63.4)$ & $216(100)$ & 0.194 & $1.15(0.53,2.50)$ \\
\hline Tertiary & $59(33.9)$ & $115(66.1)$ & $174(100)$ & $p=0.659$ & $1.03(0.47,2.26)$ \\
\hline Total & $149(32.5)$ & $274(64.8)$ & $423(100)$ & & \\
\hline \multicolumn{6}{|c|}{ Husbands employment status (ever married) $n=462$} \\
\hline Employed & $145(35.1)$ & $268(64.9)$ & $413(100)$ & 0.002 & 1.00 \\
\hline Unemployed & $4(40.0)$ & $6(60.0)$ & $10(100)$ & $P=0.747$ & $1.23(0.34,4.44)$ \\
\hline Total & $149(32.5)$ & $274(64.8)$ & $423(100)$ & & \\
\hline \multicolumn{6}{|c|}{ Currently staying together with husband (ever married) $n=423$} \\
\hline Yes & $121(34.6)$ & $229(65.4)$ & $350(100)$ & 0.232 & $0.84(0.51,1.43)$ \\
\hline No & $28(38.4)$ & $45(61.6)$ & $73(100)$ & $p=0.630$ & 1.00 \\
\hline Total & $149(32.5)$ & $274(64.8)$ & $423(100)$ & & \\
\hline \multicolumn{6}{|c|}{ Ever discussed family planning issues with partner $(n=560)$} \\
\hline Yes & $149(64.5)$ & $82(35.5)$ & $231(100)$ & 148.229 & $10.90^{*}(7.24,16.43)$ \\
\hline No & $47(14.3)$ & $282(85.7)$ & $329(100)$ & $p<0.0001^{*}$ & 1.00 \\
\hline Total & $196(35.0)$ & $364(65.0)$ & $560(100)$ & & \\
\hline \multicolumn{6}{|c|}{ Partner in favor of modern family planning $(n=560)$} \\
\hline Yes & $136(54.4)$ & $114(45.6)$ & $250(100)$ & 73.179 & $4.97^{*}(1.85,7.24)$ \\
\hline No & $60(19.4)$ & $250(80.6)$ & 310)100) & $p<0.0001^{*}$ & 1.00 \\
\hline Total & $196(35.0)$ & $364(65.0)$ & $560(100)$ & & \\
\hline \multicolumn{6}{|c|}{$\begin{array}{l}\text { Respondent can access and pay for family planning service without partner's financial support } \\
\qquad(\mathrm{n}=560)\end{array}$} \\
\hline Yes & $120(40.5)$ & $176(59.5)$ & $296(100)$ & 7.960 & $1.69^{*}(1.19,2.40)$ \\
\hline No & $76(28.8)$ & $188(71.2)$ & $265(100)$ & $p=0.005^{*}$ & 1.00 \\
\hline Total & $196(35.0)$ & $364(65.0)$ & $560(100)$ & & \\
\hline
\end{tabular}

* = statistically significant.

pay for family planning services without their husbands support, $\left(X^{2}=7.96, p=\right.$ 0.004 , OR: $1.69 ; 1.19-2.40$ ) were more likely to be using any form of contraception than their counterparts. Husbands' level of education, occupation, and current stay with husband did not play any significant role in current use of any form of family planning method, $(P>0.05)$.

\section{Discussion}

The contraceptive awareness reported in this study was high (99.1\%) and the 
common contraception methods known were; pills, injections and condoms while the least known method was traditional method. Also the level of knowledge among the respondents about contraceptive method was high $(81.1 \%)$. This was higher than the national average of $85.2 \%$ for all women of reproductive age in Nigeria but consistent with $99.7 \%$ reported from the state [9]. It was also higher than the figure reported from a Country wide survey [14]. This high awareness might likely be due to increased contraceptive information spread through the mass media and other sources. The contraceptive prevalence was $35.2 \%$, it higher than figures reported from the 2013 National survey (15.0\%), and a figure reported from urban localities of the country (27.0\%) [8] [9] [14]. It was consistent with the figure reported from the state (34.1\%) [9]. The high awareness noticed in this survey did not translate to high contraceptive use and this could have accounted for poor health indices recorded in the state and country at large. This brings to question the quality of information gotten and the source of contraceptive information. The commonly used contraceptives were condoms, Natural methods, pills and injections. This was still the pattern in some of the reviewed works [5] [9] [12] [14] even though the prevalence of tubal ligation in this study was higher than what was reported in these studies. The primary reason why condom was the commonest used was its dual purpose of prevention of unwanted pregnancy and protection from the transmission of sexually transmitted diseases which is on the rise globally.

Age of the respondents was found to influence contraceptive use significantly, both ever and current use increased with increasing age of women. Older women above 40 years of age were more likely to use than those in the younger age group. This pattern has been reported in several studies [4] [6] [14]-[19]. This pattern possibly reflects the desire for more children among young women than the older women who would have completed their families and would want to use family planning to limit family size rather than for spacing births which is commoner among women in the young age group. However an older study from Burkina Faso and New Delhi India showed that current use of contraceptive method was higher among the younger age groups, 20 - 29 years and $15-29$ years of age respectively [29] [30].

Religion influenced family planning method use with those in orthodox denominations being more likely to use family planning than others. This pattern has been reported from several studies around the world [4] [6] [15] [17] [19] [21] [22]. This supports the widely speculated belief that some religions bodies tend to have a higher disapproval rate for contraceptive method use than others. This is not a good report in a country like Nigeria with deep religious beliefs, high population growth rate and high infant and maternal mortalities. A good starting point may be advocacy to Clerics and church leaders for them to help convince their adherents that family planning is beneficial and to explore possible areas of common ground among those with dogmatic disapproval to contraception.

Fertility related issues i.e. family size and number males in the family was 
found to influence family planning method use in this study with those with large family size and number of males being more likely to use than those with smaller family sizes. This report was consistent with findings from other studies [4] [6] [17] [18] [19] [21] [26] [27], though a few reported no influence [14] [15]. This further strengthens the fact that most women use family planning to limit family size rather than space pregnancies. Our culture still shows preference for males, forcing women with no males to continue having children, irrespective of the inherent damages thereby limiting their use of contraceptives as typified in our study.

Those who earn high incomes were more likely to use than those with low income. Also those who can access and pay for family planning services without their husband's support were more likely to use than those who cannot pay without their husband financial support. Ever use was more among working women than those not working. Significant effect of socioeconomic status of women (monthly income, occupation, ownership of household items and wealth index) on contraceptive use has been reported from several studies around the world [4] [5] [6] [17] [18] [19] [20] [25], though a few other studies showed no significant relationship [14] [24]. This shows that financial issues play a vital role in whether a woman will use family planning or not. This portrays a great danger in our country which is a male dominant society where the females seem to be less empowered financially and have to depend on their husbands for support. This calls for urgency in the empowerment of women and political will to provide free family planning services that is culturally acceptable to the people.

Though educational status of the respondents was not found to influence contraceptive use in this study, a pattern which has also been reported in some other studies [4] [15] [22], yet contraceptive knowledge has high influence on contraceptive use with those having good knowledge about contraceptive methods being more likely to use than others. This pattern of influence of contraceptive knowledge on contraceptive use was consistent with findings from other studies within and outside the Country [4] [5] [23] [24] [31] [32] [33]. Despite the fact that education did not influence use, it was found that increase in educational level of respondents significant increased the level of contraceptive knowledge among the women. This has been reported in a study in Nigeria [34]. Thus whatever affects knowledge will likely influence contraceptive use, hence the right information when given properly improves knowledge and could lead to an increase in contraceptive use.

Other factors found to have positive impact on family planning in this study were issues related to male involvement or roles in family planning use. Those women who had ever discussed family planning with their partners or spouses were about 10 times more likely to use than those who have not discussed it with their spouses. This finding was similar to reports from studies elsewhere [27] [35] [36]. Also the study revealed significant association between partners support in favor of modern family planning and current contraceptive use with those whose partners were in support being about 5 times more likely to use 
than those whose partners were not. Similar studies done in different parts of the Country and beyond showed results consistent with the above findings [4] [15] [23] [27] [28] [31] [37] [38]. This finding corroborates the fact that most of the Nigerian society is patrilineal with strong male influence on many household decisions making including those involving reproductive health matters. This is of a serious public health concern that needs urgent attention even as majority of the women $(92.7 \%)$ reported that their husband's opinion is important in family planning matters. Despite this perceived importance of males in family planning use in this study, some studies reported unfavorable attitude of spouses towards the use of family planning [39] [40] [41].

Limitation to study: The study was a cross-sectional study and the findings were based on the responses of the study participants which may be subject to some level of response bias. Thus care should be taken in generalizing the findings of this study. Also it will be difficult to establish cause effect relationship.

\section{Conclusion}

Despite a high awareness found in this study, the use of family planning method was low. The factors influencing contraceptive use as found in this study were: age of women, religion, family size, number of males, monthly income, contraceptive knowledge, ability to access and pay for family planning services without partner financial support, prior discussion of family planning with partner and partner being in support of modern family planning methods. Most of these factors are modifiable and serious interventions focused on proper health education, provision of free and accessible family planning services, provision of incentives, inclusion of men in family planning and implementing of the existing family planning policies in the country will go a long way in improving the utilization of family planning services. These interventions among others have shown significant improvement in family planning services utilizations in countries where they have been implemented.

\section{Acknowledgements}

We want to thank these women who participated in this study including the Community Leaders who gave us access to their communities. This work is part of a postgraduate thesis submitted for the award of Fellowship of the West African College of Physicians (FWACP).

\section{Authors Contribution}

$\mathrm{CBD}$ carried out the research from conception to the write up of the final draft of the article. OE had supervised and critically commented at each stage of the research. All the other authors read and approved the final manuscript.

\section{Competing Interest}

The authors hereby declare no competing interests. 


\section{Source of finding}

There was no external source of funding.

\section{References}

[1] World Health Organization (1971) Evaluation of Family Planning. Techn. Rep. Series, WHO, Geneva, No. 483, 25-66.

[2] World Health Organisation (1970) Evaluation of Family Planning Services. Techn. Rep. Series, WHO, Geneva, No. 2422, 1-3.

[3] Oyedokun, A.O. (2007) Determinants of Contraceptive Usage Lessons from Women in Osun State. Nigeria Journal of Humanities and Social Science, 1, 1-6.

[4] Okech, T.C., Wawire, N.W. and Mburu, T.K. (2011) Contraceptive Use among Women of Reproductive Age in Kenya's City Slums. International Journal of Business and Social Science, 2, 22-43.

[5] Ibnouf, A.H., Vanden Borne, H.W. and Maarse, J.A.M. (2007) Utilization of Family Planning Services by Married Sudanese Woman of Reproductive Age. Eastern Mediterranean Health Journal, 13, 1372-1381. https://doi.org/10.26719/2007.13.6.1372

[6] Sharma, V., Mohan, U., Das, V. and Awasthi, S. (2012) Socio-Demographic Determinants and Knowledge, Attitude, Practice: Survey of Family Planning. Journal of Family Medicine and Primary Care, 1, 43-47. https://doi.org/10.4103/2249-4863.94451

[7] Population Reference Bureau (2013) World Population Data Sheet, 2013. UNDP, Washington DC. http://www.prb.org

[8] Population Reference Bureau (2015) The Urban-Rural Divide in Health and Development Data Sheet. UNDP, Washington DC, 11-12. http://www.prb.org

[9] National Population Commission (NPC), Nigeria and ICF Macro International (2014) Nigerian Demographic and Health Survey 2013. NPC and ICF International, Abuja, Rockville.

[10] United Nations Economic Commission for Africa (2013) Report on the Progress in Achieving the Millennium Development Goals in Africa. United Nations Economic Commission for African, Addis Ababa.

[11] National Population Commission (NPC) Nigeria and ICF Macro International (2003) Nigerian Demographic and Health Survey. NPC and ICF International, Abuja, Rockville.

[12] National Population Commission (NPC) Nigeria and ICF Macro International (3008) Nigeria Demographic and Health Survey. NPC and ICF International, Abuja, Rockville.

[13] National Population Commission (NPC), Nigeria (2004) National Policy on Population for Sustainable Development. National Population Commission, Abuja.

[14] Oye-Adeniran, B.A., Adewole, I.F., Umoh, A.V., Oladokun, A., Gbadegesin, A., Ekanem, E.E., Yusuf, B., Odeyemi, K.O., Iwere, N. and Mahmoud, P. (2006) Community Based Study of Contraceptive Behaviour in Nigeria. African Journal of Reproductive Health, 10, 90-104. https://doi.org/10.2307/30032462

[15] Kana, M.A., Tagurum, Y.O., Hassan, Z.I., Afolanranmi, T.O., Ogbeyi, G.O., Difa, J.A., et al. (2016) Prevalence and Determinants of Contraceptive Use in Rural North Eastern Nigeria: Result of a Mixed Qualitative and Quantitative Assessment. Annals of Nigerian Medicine, 10, 3-10. https://doi.org/10.4103/0331-3131.189801 
[16] Agbo, H.A., Ogbonna, C. and Okeahialam, B.N. (2013) Factors Related to the Uptake of Contraceptive in a Rural Community in Plateau State, Nigeria: A Cross-Sectional Community Study. Journal of Medicine in the Tropics, 15, 107-112. https://doi.org/10.4103/2276-7096.123583

[17] Hafez, A.A.A. (2014) Factors Affecting the Family Planning Methods Used by Currently Married Women in Rural Egypt. American Journal of Research Communication, 2, 324-341.

[18] Shaikh, S. and Dwivedi, S. (2014) Predictors of Family Planning Methods among Women of Allahabad District, Uttar Pradesh. Health and Population in Perceptive and Issues, 37, 118-127.

[19] Igbodekwe, F.C., Oladimeji, O., Oladimeji, K.E., Adeoye, I.A., Akpa, M.O. and Lawson, L. (2014) Utilization of Modern Contraceptive among Women of Child Bearing Age in Resource Constraint Setting: Evidence from 2008 National Demographic and Health Survey in Nigeria. Journal of Health Science, 4, 72-78.

[20] Alenoghena, I.O., Isah, E.C., Isara, A.R., Ameh, S.S. and Adam, V.Y. (2015) Uptake of Family Services among Women of Reproductive Age in Edo North Senatorial District, Edo State, Nigeria. Sub-Saharan African Journal of Medicine, 2, 154-159. https://doi.org/10.4103/2384-5147.172433

[21] Envuladu, E.A., Agbo, H.A., Mohammed, A., Chia, L., Kigbu, J.H. and Zoakah, A.I. (2012) Utilization of Modern Contraceptives among Female Traders in Jos South LGA of Plateau State, Nigeria. International Journal of Medicine and Biomedical Research, 1, 224-231. https://doi.org/10.14194/ijmbr.1310

[22] Olugbenga-Bello, A.I., Abodunrin, O.L. and Adeomi, A.A. (2011) Contraceptive Practices among Women in Rural Communities in South-Western Nigeria. Global Journal of Medical Research, 11, 1.

[23] Mubita-Ngoma, C. and Kadantu, M.C. (2011) Knowledge and Use of Modern Family Planning by Rural Women in Zambia. Curationis, 33, 17-22.

[24] Amentie, M., Abera, M. and Abdulahi, M. (2015) Utilization of Family Planning Services and Influencing Factors among Women of Child Bearing Age in Assosa District, Benishangul Gumuz Regional State, West Ethiopia. Science Journal of Clinical Medicine, 4, 52-59. https://doi.org/10.11648/j.sjcm.20150403.11

[25] Abiodun, O.P., Usman, S.O., Olomide, J., Hamza, S. and Adesina, A. (2016) Contraceptive Practices in a South-Western Nigerian Urban Population. International Journal of Health Sciences and Research, 6, 264-269.

[26] Apanga, P.A. and Adam, M.A. (2015) Factors Influencing the Uptake of Family Planning Services in the Talensi District, Ghana. Pan African Medical Journal, 20, 10. https://doi.org/10.11604/pamj.2015.20.10.5301

[27] Mekonnem, W. and Worku, A. (2011) Determinants of Low Family Planning Use and High Unmet Need in Butajira District, South Central Ethiopia. Reproductive Health, 8, 37. https://doi.org/10.1186/1742-4755-8-37

[28] Raji, M.O., Oche, M.O., Kaoje, A.U., Raji, H.O. and Anglo, J.T. (2013) Awareness and Utilization of Family Planning Commodities in a Rural Community of North West, Nigeria. Caliphate Medical Journal, 1, 103-104.

[29] McGinn, T., Sebgo, P., Fenn, T. and Bamba, A. (1987) Family Planning in Burkina Faso: Results of Survey. Studies in Family Planning, 20, 325-331. https://doi.org/10.2307/1966435

[30] Reddy, P.J. (1984) Differential Contraceptive Use among the Slum and Non-Slum Dwellers; A Study of Hydradad City. Health and Population Perspectives and Users, 7, 115-128. 
[31] Fantahun, M. (2006) Comparative Study of the Characteristics of Family Planning Service Users and Non-Users in North West Ethiopia. African Journal of Reproductive Health, 10, 62-71. https://doi.org/10.2307/30032444

[32] Smith, J., McFedyen, L., Harrison, A. and Zuma, K. (2002) Where Is the Condom: Contraceptive Practice in a Rural District of South Africa. African Journal of Reproductive Health, 6, 71-78. https://doi.org/10.2307/3583132

[33] Mannan, H.R. (2002) Factors in Contraceptive Method Choice, Bangladesh: Goal-Competence Evaluation and Access. Advances in Contraception, 65, 337-364. https://doi.org/10.1016/S0010-7824(01)00303-1

[34] Olaseha, O.I., Ajuwon, A.J. and Onyejekwe, O.C. (2004) Reproductive Health Knowledge and Use of Contraceptive among Adolescent Mothers in a Sub-Urban Community in Oyo State, Nigeria. The African Journal of Medical Sciences, 33, 139-143.

[35] Sharan, M. and Valente. T.W. (2002) Spousal Communication and Family Planning Adoption: Effects of a Radio Drama Serial in Nepal. International Family Planning Perspectives, 28, 16-25. https://doi.org/10.2307/3088271

[36] Lasee, A. and Becker, S. (1997) Husband-Wife Communication about Family Planning and Contraceptive Use in Kenya. International Family Planning Perspectives, 23, 15-20. https://doi.org/10.2307/2950781

[37] Beekle, A.T. and McCabe, C. (2006) Awareness and Determinants of Family Planning Practice in Jimma, Ethiopia. International Nursing Review, 53, 269-276. https://doi.org/10.1111/j.1466-7657.2006.00492.x

[38] Fitaw, Y., Berhane, Y. and Worku, A. (2004) Impact of Child Mortality and Fertility Preferences on Fertility Status in Rural Ethiopia. East African Medical Journal, 81, 300-306. https://doi.org/10.4314/eamj.v81i6.9179

[39] Chadwick, N., Dhillon, B.S., Kumbo, I. and Saxena, N.C. (2003) Contraceptive Knowledge, Practice, and Utilization of Services in the Rural Areas of India. Indian Journal of Medical Sciences, 57, 303-310.

[40] Khan, M.A. (1996) Factors Affecting the Use of Contraceptives in Matlab, Bangladesh. Journal of Biosocial Science, 28, 264-279. https://doi.org/10.1017/S0021932000022343

[41] Tehrani, F.R., Farahani, F.K. and Hashemi, A. (2001) Factors Influencing Contraceptive Use in Teheran. Family Planning Practices, 18, 204-208. https://doi.org/10.1093/fampra/18.2.204 